\title{
Inhibition of Nitric Oxide Synthesis and Gene Knockout of Neuronal Nitric Oxide Synthase Impaired Adaptation of Mouse Optokinetic Response Eye Movements
}

\author{
Akira Katoh, 1,3,4 Hiromasa Kitazawa, ${ }^{2,3}$ Shigeyoshi Itohara, 1,3,5 and Soichi Nagao ${ }^{2,3}$ \\ ${ }^{1}$ Laboratory for Behavioral Genetics, Brain Science Institute (BSI), RIKEN, Wako, Saitama 351-0198, Japan; ${ }^{2}$ Department of Physiology, \\ Jichi Medical School, Minamikawachi, Tochigi 329-0498, Japan; ${ }^{3}$ CREST, JST, Saitama 332-0012, Japan
}

\begin{abstract}
Nitric oxide (NO) plays a key role in synaptic transmission efficiency in the central nervous system. To gain an insight on the role of NO in cerebellar functions, we, here, measured the dynamics of the horizontal optokinetic response (HOKR) and vestibulo-ocular reflex (HVOR), and the adaptation of HOKR in mice locally injected with $N^{\mathrm{G}}$-monomethyl-L-arginine (L-NMMA) that inhibits NO synthesis and in mice devoid of neuronal nitric oxide synthase (nNOS). Local application of L-NMMA into the cerebellar flocculi induced no change in the dynamics of the HOKR but markedly depressed the adaptation of the HOKR induced by 1 hr of sustained screen oscillation. A slight difference was seen in the HOKR but not in the HVOR dynamics between $n N O S^{-/-}$ mutant and wild-type mice. One hour of sustained screen oscillation induced adaptation of the HOKR gains in wild-type mice but not in mutants. These observations suggest that NO is essential for the adaptation of the HOKR and that nNOS is the major enzyme for NO synthesis in the process.
\end{abstract}

Nitric oxide (NO) plays a number of important roles as a biological messenger molecule. In the central nervous system, the importance of NO in neural plasticity has recently been recognized. In the cerebellum, conjunctive stimuli to parallel and climbing fibers evoke long-term depression (LTD) of synaptic transmission of parallel fiber-Purkinje cell synapses (Ito et al. 1982; Ekerot and Kano 1985). The role of NO in induction of cerebellar LTD was first suggested by Ito and Karachot (1990) and Crepel and Jaillard (1990), based on findings that the application of $N^{\mathrm{G}}$-monomethyl-Larginine (L-NMMA), an inhibitor of NO synthesis, or hemoglobin, an NO scavenger, prevented induction of LTD in cerebellar slices. Furthermore, release of endogenous NO was observed at induction of cerebellar LTD (Shibuki and Okada 1991). It has also been demonstrated that photorelease of caged NO inside the Purkinje cell with either depolarization or uncaged $\left[\mathrm{Ca}^{2}{ }^{+}\right]_{i}$ is sufficient to induce LTD in cerebellar slices (Lev-Ram et al. 1995, 1997a), further supporting the role of NO in cerebellar LTD.

$\mathrm{NO}$ is produced from arginine by nitric oxide synthase (NOS). Three major isoforms of NOS have been identified: neuronal NOS (nNOS), endothelial NOS (eNOS), and induc-

${ }^{4}$ Present address: Department of Biophysics, Kyoto University, Sakyo-ku, Kyoto 606-8502, Japan.

${ }^{5}$ Corresponding author.

E-MAIL sitohara@brain.riken.go.jp; FAX 81-48-467-9725. ible NOS (iNOS). nNOS is prominent in neurons and particularly abundant in the granule cells in the cerebellum (Knowles and Moncada 1994; Kobzik et al. 1994; Rodrigo et al. 1994). eNOS is also expressed in the granule cells at a lesser level than nNOS (Dinerman 1994). Because Lev-Ram et al. (1997b) observed no signs of LTD in whole-cell patch clamped Purkinje cells in cerebellar slices obtained from $n \mathrm{NOS}^{-/-}$mutant mice, nNOS appears to be the major source of NO essential for LTD in cerebellar slice. However, it has not been established whether the deficits of LTD link to anomalies in motor controls mediated by the cerebellum.

Marr (1969) and Albus (1971) proposed a synaptic plasticity as a cellular mechanism of learning in the cerebellum. Ito (1989) suggests that cerebellar LTD underlies the cerebellar-dependent motor learning. Previous studies have shown that applications of either an inhibitor for NO synthesis or NO scavenger, which block cerebellar LTD, impair some forms of motor learning, such as adaptation of the horizontal vestibulo-ocular reflex (HVOR) (Nagao and Ito 1991; Li et al. 1995), smooth pursuit eye movement (Nagao and Kitazawa 2000), coordinated locomotion (Yanagihara and Kondo 1996), and eyeblink conditioning (Chapman et al. 1992). Recently, we developed a method for measuring the horizontal optokinetic response (HOKR) and HVOR eye movements and an adaptation paradigm for the HOKR in mice (Katoh et al. 1998). We revealed here by local appli-

LEARNING \& MEMORY 7:220-226 @ 2000 by Cold Spring Harbor Laboratory Press ISSN1072-0502/00 \$5.00

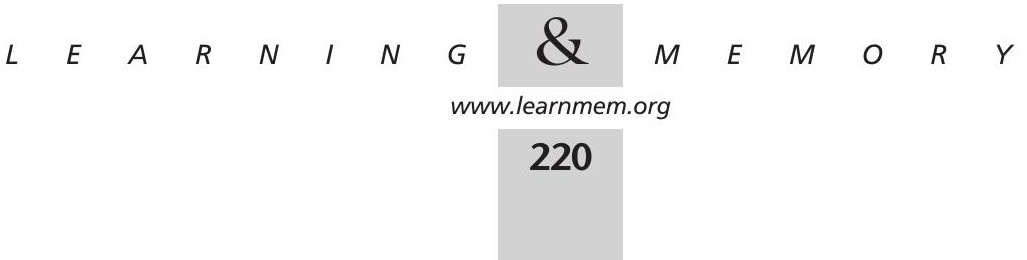


cation of L-NMMA that NO-dependent cascades in the flocculus underlie the adaptation of the HOKR, and we observed the impaired adaptation of the HOKR in $n \mathrm{NOS}^{-/-}$ mutant mice. These results from both pharmacological and genetical methods consistently indicate the important role of NO in the adaptation of the HOKR and of nNOS in cerebellar functions.

\section{RESULTS}

\section{Local Application of L-NMMA Impaired the Adaptation of the HOKR}

To assess the role of cerebellar NO for the adaptation of the HOKR, we applied either $0.5 \mu \mathrm{l}$ of $20 \mathrm{~mm}$ L-NMMA, a specific NO synthesis blocker, or the same amount of $20 \mathrm{~mm}$ D-NMMA, an inactive isomer of L-NMMA, to the flocculi of C57BL/6 mice via the paraflocculi. To confirm the diffusibility of chemicals and reproducibility of the applications, $0.5 \mu \mathrm{l}$ of $1 \%$ FITC/PBS ( $\mathrm{pH} 7.4$ ) was injected into four C57BL/6 mice in the same manner as D- or L-NMMA/PBS ( $\mathrm{pH}$ 7.4). FITC reproducibly diffused over the flocculus in this protocol. In three of four mice, FITC diffused over nearly whole areas of flocculi bilaterally. In the remaining one mouse, FITC diffused over nearly the whole area of the right flocculus and about a two-thirds area of the left flocculus. A representative result is shown in Figure 5, B and C. To see the effects of these chemical application on the dynamics of eye movement (meaning multiple measures of gains and phases at different screen velocities), we measured the HOKR, because the HOKR dynamics were stable during 1-hr restraining in the apparatus (Katoh et al. 1998). No differences were seen in the HOKR after applications of 20 mм D-NMMA or L-NMMA (Fig. 1A,B). The phase lags of the HOKR were very small $\left(+2^{\circ}\right.$ to $\left.-2^{\circ}\right)$ and not altered by applications of D-NMMA and L-NMMA. Therefore, the applications of either D-NMMA or L-NMMA little affected the HOKR dynamics. Mice bilaterally injected with $20 \mathrm{~mm}$ DNMMA were exposed to $1 \mathrm{hr}$ of sustained screen oscillation by $15^{\circ}$ at $0.17 \mathrm{~Hz}\left(7.9^{\circ} / \mathrm{sec}\right)$, which induced significant increase in the HOKR gain from $0.39 \pm 0.02$ to $0.45 \pm 0.03$ (mean \pm s.E., $n=12, P<0.005$ by Student's $t$-test) (Fig. $2 \mathrm{~B}$ ). After 2 days, we injected $20 \mathrm{~mm}$ L-NMMA bilaterally and exposed them to $1 \mathrm{hr}$ of sustained screen oscillation. The HOKR gains remained unchanged from $0.41 \pm 0.02$ to $0.42 \pm 0.02$ ( $n=12, P>0.80$ by Student's $t$-test; Fig. $2 \mathrm{~B})$. After 2 days, these mice were again exposed to $1 \mathrm{hr}$ of sustained screen oscillation. These mice showed significant increase in the HOKR gain from $0.39 \pm 0.02$ to $0.50 \pm 0.03$ ( $n=12, P<0.005$ by Student's $t$-test) in this recovery test (Fig. 2B), which indicates that these mice did not receive chronic tissue damage by multiple insertion of injection needles. Thus, the data from these pharmacological experiments suggest that cerebellar NO is essential for the adaptation of the HOKR.
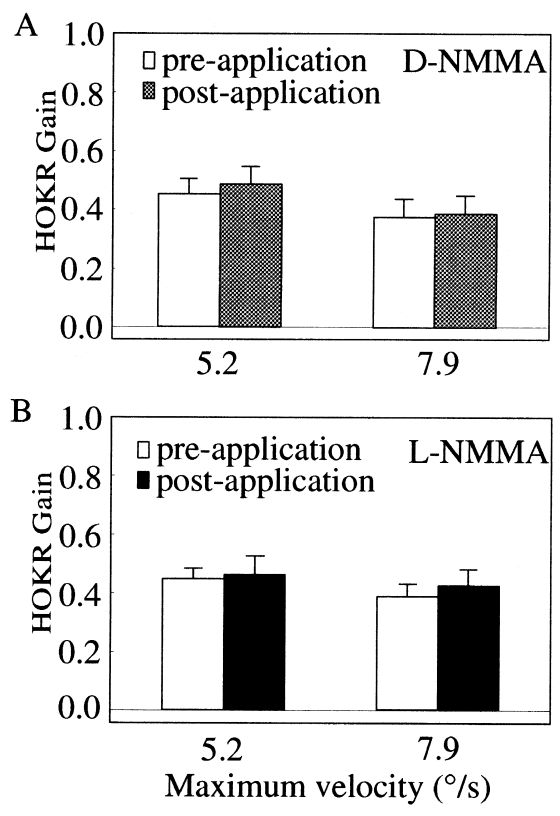

Figure 1 D-NMMA or L-NMMA applications do not alter the HOKR gains. The HOKR gains before and after application of $D$ NMMA $(A)$ and L-NMMA $(B)$. The HOKR was measured by sinusoidal oscillation of the checked-pattern screen (square size $4^{\circ}$ ) by $10^{\circ}$ peak to peak at $0.17 \mathrm{~Hz}$ (maximum velocity, $5.2^{\circ} / \mathrm{sec}$ ) and $15^{\circ}$ at $0.17 \mathrm{~Hz}\left(7.9^{\circ} / \mathrm{sec}\right)$. No difference was seen in the dynamic characteristics of the HOKR before and after application of chemicals. Vertical bars indicate standard errors of means in all panels.

\section{$n$ NOS $^{-/-}$Mutant Mice Revealed an Impaired Adaptation of the HOKR}

We then analyzed $n \mathrm{NOS}^{-/}$mutant mice, to examine whether nNOS is the major source for cerebellar NO. As shown in Figure 3A, the HOKR gains of mutant mice were almost the same as those of wild-type mice at $2.6^{\circ} / \mathrm{sec}$, $3.5 \% \mathrm{sec}$, and $5.2 \% \mathrm{sec}$ of maximum screen velocities but larger than that of wild-type mice by 0.09 only at $10.5 \%$ sec ( $P<0.05$ by Student's $t$-test). Concomitantly, the lags of the HOKR phases in mutant mice were smaller than those in wild-type mice by $2.0^{\circ}-4.6^{\circ}$ at all screen velocities ( $P<0.005$ by two-way ANOVA; Fig. 3B). On the contrary, as shown in Figure 3, C and D, the HVOR gains and phases were similar ( $P>0.10$ by two-way ANOVA) and showed a stimulus frequency dependency in both wild-type and mutant mice. Stimulus frequency dependency of the HVOR has been reported by De Zeeuw et al. (1998). The HVOR gains and phases in this study were different from those of C57BL/6 mice reported previously (Katoh et al. 1998). This may be due to the effects of visual inputs derived from the checked-pattern screen placed around the turntable during the measurements of the HVOR. Even under dark conditions, small visual inputs from the stationary screen could modulate the HVOR. This is the reason why the high gains and low phase lags were observed in the HVOR in our previous study. In the present study, we replaced the

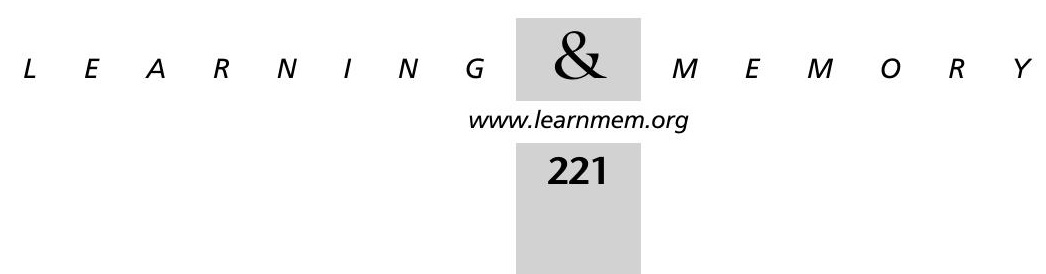


A
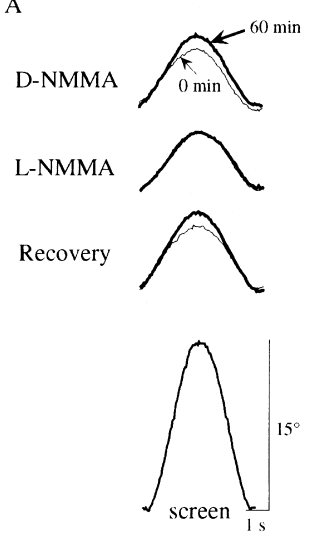

B

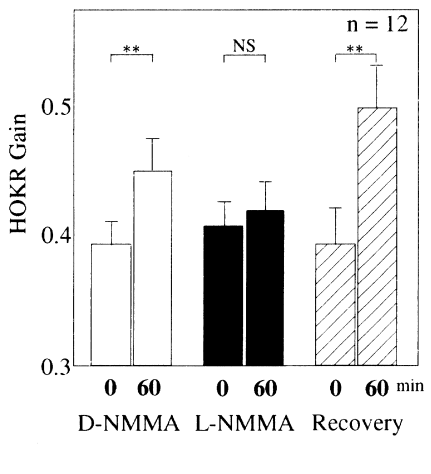

Figure 2 Impairment of adaptation of the HOKR by L-NMMA local application. (A) Examples of eye movements during D-NMMA, L-NMMA, and the recovery test in the case of one $\mathrm{C} 57 \mathrm{BL} / 6$ mouse. Each trace is the average of the evoked eye movements $>12$ cycles. Plain and bold lines indicate the averaged eye position traces before and after sustained screen oscillation for 60 min, respectively. In this example, the HOKR gains changed from 0.40 to 0.48 in D-NMMA, from 0.41 to 0.40 in L-NMMA, and from 0.43 to 0.53 in the recovery test. The averaged screen position trace is shown at bottom. (B) Comparison of HOKR gain among D-NMMA, L-NMMA, and the recovery test. Averaged data obtained from 12 different mice were indicated. The HOKR gains in these tests were compared before and after $1 \mathrm{hr}$ of sustained screen oscillation by $15^{\circ}$ at $0.17 \mathrm{~Hz}$. Note that the adaptation of the HOKR was abolished by L-NMMA but not by D-NMMA and not in the recovery test. $\left.{ }^{* *}\right) P<0.005$; (NS) not significant by Student's $t$-test.

checked-pattern screen to a black monotone screen and paid much attention to eliminating the artifacts from such small visual inputs during the measurement of the HVOR.

The adaptability of HOKR was tested at $10^{\circ}$ and 0.17 $\mathrm{Hz}\left(5.2^{\circ} / \mathrm{sec}\right)$ of screen oscillation. At this screen velocity, no difference was seen in the HOKR gain measured just before sustained screen oscillation between mutant $(0.48 \pm 0.01, n=12)$ and wild-type $(0.48 \pm 0.01, n=8)$ mice. One hour of sustained screen oscillation significantly increased the HOKR gain in wild-type control littermates from $0.48 \pm 0.01$ to $0.55 \pm 0.02(n=8, P<0.005$ by Student's $t$-test) (Fig. 4A,B). However, $1 \mathrm{hr}$ of sustained screen oscillation affected little on the HOKR gain in mutant mice from $0.48 \pm 0.01$ to $0.51 \pm 0.02(n=12, P>0.05$ by Student's $t$-test) (Fig. 4A,B). Thus, the adaptation of the HOKR was impaired in $\mathrm{nNOS}^{-/-}$mutant mice.

\section{Estimation of Retinal Slips Necessary for the Adaptation of the HOKR in Mice}

Our previous study showed that $1 \mathrm{hr}$ of sustained screen oscillation by $10^{\circ}$ at $0.17 \mathrm{~Hz}$ (maximum screen velocity; $5.2^{\circ} / \mathrm{sec}$ ) increased the HOKR gain, whereas those by $5^{\circ}$ at $0.11 \mathrm{~Hz}\left(1.7^{\circ} / \mathrm{sec}\right)$ did not affect the HOKR gain in C57BL/6 mice (Katoh et al. 1998). Furthermore, in this study, $1 \mathrm{hr}$ of sustained screen oscillation by both $10^{\circ}$ at $0.17 \mathrm{~Hz}$ (Fig. 4,
A

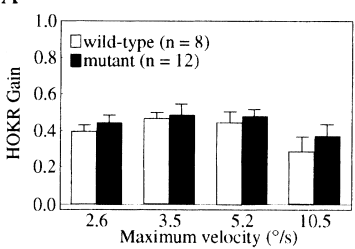

C

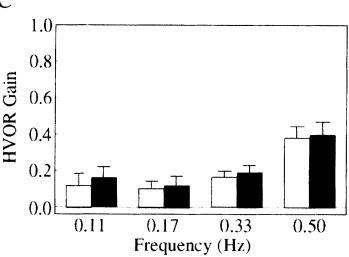

B
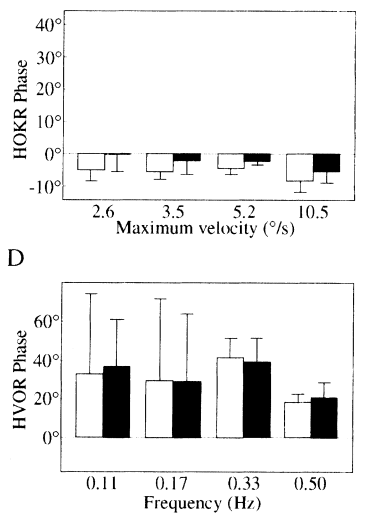

Figure 3 Dynamic characteristics of HOKR and HVOR in $n \mathrm{NOS}^{-1-}$ mutant mice. The gain $(A)$ and phase $(B)$ of HOKR were measured by sinusoidal oscillation of the checked-pattern screen. The screen was oscillated by $5^{\circ}$ peak to peak at $0.17 \mathrm{~Hz}$ (maximum velocity, $\left.2.6^{\circ} / \mathrm{sec}\right), 10^{\circ}$ at $0.11 \mathrm{~Hz}\left(3.5^{\circ} / \mathrm{sec}\right), 10^{\circ}$ at $0.17 \mathrm{~Hz}\left(5.2^{\circ}\right.$ $\mathrm{sec})$, and $20^{\circ}$ at $0.17 \mathrm{~Hz}\left(10.5^{\circ} / \mathrm{sec}\right)$. The HOKR gains of mutant mice were almost the same as those of wild-type mice at $2.6 \% \mathrm{sec}$, $3.5 \% \mathrm{sec}$, and $5.2 \% \mathrm{sec}$ of maximum screen velocities and larger than that of wild-type mice by 0.09 only at $10.5 \%$ sec $(P<0.05$ by Student's $t$-test), and the HOKR phase lags in mutant mice were smaller than those in wild-type mice at all screen velocities $(P<0.005$ by two-way ANOVA). The gain $(C)$ and phase $(D)$ of HVOR were measured by sinusoidal oscillation of the turntable in darkness. No difference was seen between wild-type and mutant mice.

data of the wild-type mice) and $15^{\circ}$ at $0.17 \mathrm{~Hz}\left(7.9^{\circ} / \mathrm{sec}\right)$ (Fig. 2, D-NMMA application and recovery test) increased the HOKR gain. The maximum retinal slip velocities during

A

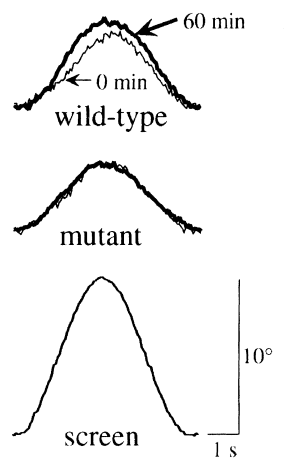

B

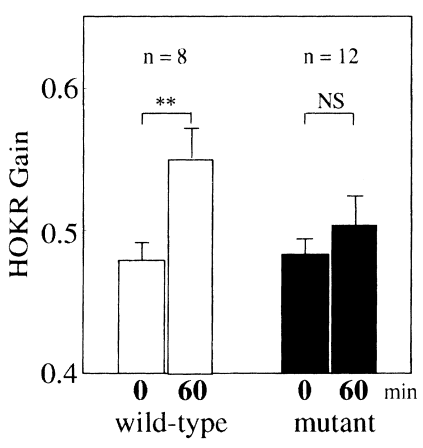

Figure 4 Impaired adaptation of the $\mathrm{HOKR}$ in $n \mathrm{NOS}^{-/-}$mutant mice. (A) Examples of averaged eye position traces of wild-type and mutant mice before and after $1 \mathrm{hr}$ of sustained screen oscillation by $10^{\circ}$ at $0.17 \mathrm{~Hz}$. Each trace is the average of the evoked eye movements $>8$ cycles. In this example, the HOKR gains changed from 0.46 to 0.54 in wild-type and from 0.48 to 0.49 in mutant mice. The averaged screen position trace is shown at bottom. (B) The HOKR gains of wild-type and mutant mice before and after 1 $\mathrm{hr}$ of sustained screen oscillation by $10^{\circ}$ at $0.17 \mathrm{~Hz}$. Whereas wild-type mice showed a significant increase in the HOKR gain by $1 \mathrm{hr}$ of sustained screen oscillation, mutant mice did not. $(* *)$ $P<0.005 ;(N S)$ not significant by Student's $t$-test.

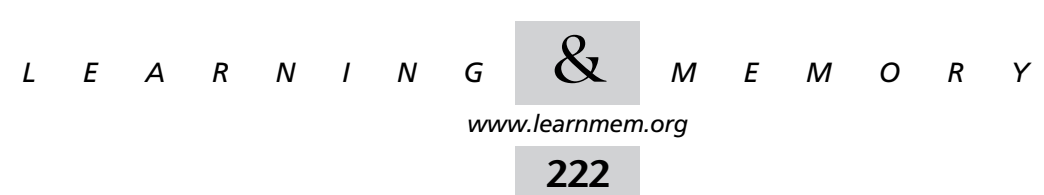


the screen oscillation were calculated as the difference between the maximum screen and eye velocity, that is, (maximum screen velocity) $\times \sqrt{1-2 G \cos \theta+G^{2}}(G$, HOKR gain; $\theta$, HOKR phase). As shown in Table 1 , sufficient retinal slips are necessary for the induction of the adaptation of the HOKR in mice, consistent with other animals. The threshold sufficient to induce the adaptation of the HOKR might be between $1.9^{\circ} / \mathrm{sec}$ and $2.7^{\circ} / \mathrm{sec}$ in the maximum retinal slip velocity. In this study, $n \mathrm{NOS}^{-/-}$mutant and wild-type control mice were exposed to the sustained screen oscillation by $10^{\circ}$ at $0.17 \mathrm{~Hz}$. At this condition, maximum retinal slip velocities were calculated as $2.7 \pm 0.1^{\circ} / \mathrm{s}$ in both genetic groups.

\section{DISCUSSION}

In the present study, we observed that the adaptation of the HOKR induced by $1 \mathrm{hr}$ of sustained screen ocillation was markedly affected by application of the NO synthesis inhibitor (L-NMMA) in the flocculus. The role of NO in cerebellar functions has been evaluated by various adaptation paradigms in many animal species by pharmacological methods. The adaptation of the HVOR was blocked by local application of hemoglobin in the subdural space of the flocculus of rabbit and monkey (Nagao and Ito 1991), and by local application of L-NMMA in the flocculus of goldfish (Li et al. 1995). Adaptation of monkey smooth pursuit eye movement was very much depressed by application of L-NMMA or hemoglobin in the subdural space over the parafloccular area (Nagao and Kitazawa 2000). Furthermore, the adaptation of stepping to perturbation of the velocity of the treadmill in cats was abolished by application of L-NMMA to the cerebellar cortical locomotion area (Yanagihara and Kondo 1996). Similarly, rabbits given L-NMMA intraperitoneally demonstrated learning deficits in eyeblink conditioning (Chapman et al. 1992). It is notable that these previous studies consistently indicate that application of these inhibitors of NO synthesis or NO scavenger markedly depressed the adaptation, but very little affected the dynamics themselves. Our study also revealed that inhibition of NO syn- thesis affected the HOKR dynamics very little but abolished the adaptation of the HOKR (Figs. 1 and 2). The results of our pharmacological study are consistent with previous studies and strongly suggest that NO synthesized in the flocculus plays an essential role for the adaptation of the HOKR in mice.

As possible sources of $\mathrm{NO}$ in the central nervous system, three isoforms of NOS, that is, nNOS, eNOS, and iNOS, have been identified. In the cerebellum, nNOS is expressed in the granule cells, basket cells (Knowles and Moncada 1994; Kobzik et al. 1994; Rodrigo et al. 1994) and Bergmann glia (Schmidt et al. 1992). eNOS is expressed mainly in the blood vessels and scarcely in the granule cells (Dinerman et al. 1994). The expression of iNOS seems to be very small in normal cerebellum, because iNOS is induced in the glial cells only after noxious stimuli (Murphy et al. 1993). So, the possible source of NO essential for the adaptation of the HOKR in the flocculus is nNOS and/or eNOS. In the present study, $n \mathrm{NOS}^{-1-}$ mutant mice actually exhibited very small gain changes of the HOKR during $1 \mathrm{hr}$ of sustained screen oscillation that induced the adaptation of the HOKR in control littermates. Thus, nNOS appears to be a major source responsible for NO-dependent adaptation of the HOKR.

Unlike in pharmacological studies, we observed subtle changes in the HOKR dynamics in $\mathrm{nNOS}^{-/-}$mutant mice. The HOKR gains of mutant mice were slightly larger and the lags of HOKR phases were smaller than those of wild-type mice. On the other hand, no differences were seen in the HVOR dynamics between mutant and wild-type mice (Fig. $3)$. The HVOR shares its neural circuitry with the HOKR both in the flocculus and vestibular nuclei. The optokinetic signals used to evoke the HOKR are mediated through the retina and accessory visual system, for example, the nucleus of the optic tract and the nucleus reticularis tegmenti pontis, to the flocculus and vestibular nuclear complex involving nucleus prepositus hypoglossi (Ito 1984). Furthermore, lesion of the flocculus did not affect the HOKR dynamics in mice (Katoh et al. 1998). So, a possibility is suggested that gene targeting of $n N O S$ may induce subtle changes in these

Table 1. A Relationship Between the Maximum Retinal Slip Velocities and the Induction of the Adaptation of the HOKR in Mice

\begin{tabular}{|c|c|c|c|c|c|}
\hline & \multirow{2}{*}{$\begin{array}{c}\text { Maximum screen velocity } \\
(\% / \mathrm{sec})\end{array}$} & \multicolumn{2}{|c|}{ HOKR } & \multirow{2}{*}{$\begin{array}{c}\text { Maximum retinal slip velocity } \\
(\% / \mathrm{sec})\end{array}$} & \multirow[b]{2}{*}{ Adaptation } \\
\hline & & gain & phase $\left(^{\circ}\right)$ & & \\
\hline \multicolumn{6}{|l|}{ C57BL/6 } \\
\hline & 1.7 & $0.64 \pm 0.03$ & $-3.3 \pm 0.8$ & $1.9 \pm 0.2$ & not induced \\
\hline & 5.2 & $0.45 \pm 0.04$ & $0.4 \pm 1.5$ & $2.8 \pm 0.1$ & induced \\
\hline & 7.9 & $0.38 \pm 0.02$ & $-2.1 \pm 0.8$ & $4.9 \pm 0.1$ & induced \\
\hline$n \mathrm{NOS}^{+/+}$ & 5.2 & $0.48 \pm 0.01$ & $-1.8 \pm 0.8$ & $2.7 \pm 0.1$ & induced \\
\hline$n N O S^{-1-}$ & 5.2 & $0.48 \pm 0.01$ & $-0.5 \pm 1.5$ & $2.7 \pm 0.1$ & not induced \\
\hline
\end{tabular}

Maximum screen velocities are $1.7 \% \mathrm{sec}\left(5^{\circ}\right.$ at $\left.0.11 \mathrm{~Hz}\right), 5.2^{\circ} / \mathrm{sec}\left(10^{\circ}\right.$ at $\left.0.17 \mathrm{~Hz}\right)$, and $7.9^{\circ} / \mathrm{sec}\left(15^{\circ}\right.$ at $\left.0.17 \mathrm{~Hz}\right)$. The data at $1.7 \% / \mathrm{sec}$ and $5.2 \%$ sec in C57BL/6 were referred to our previous study (Katoh et al. 1998). Maximum retinal slip velocities during the screen oscillation were calculated as (maximum screen velocity) $\times \sqrt{1-2 G \cos \theta+G^{2}}(G$, HOKR gain; $\theta$, HOKR phase).

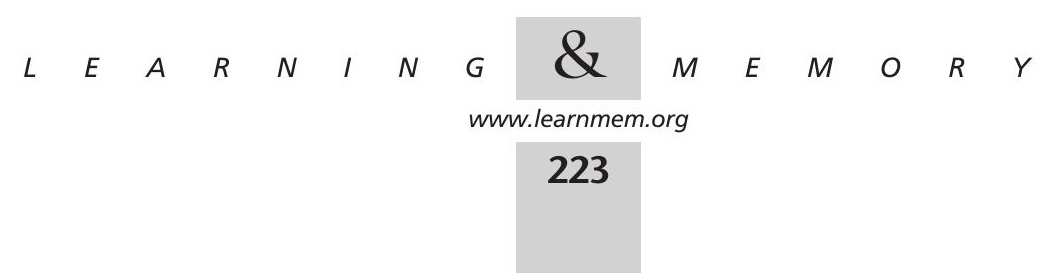


precerebellar visual areas and cause slight changes in the HOKR dynamics in $\mathrm{nNOS}^{-/-}$mutant mice. One can argue that the impaired adaptation of the HOKR in $\mathrm{nNOS}^{-/-}$mutant mice may just reflect developmental abnormalities in the precerebellar circuitry but not the function of the flocculus. However, this is unlikely because of the following reasons: The difference of the HOKR gain between $n \mathrm{NOS}^{-/-}$mutant and wild-type mice was apparent at only the highest screen velocity examined in this study $(10.5 \%$ sec). It is notable that the gain difference in the HOKR dynamics between two groups was negligibly small at the condition used for the adaptation of the HOKR (screen velocity, $5.2^{\circ} / \mathrm{sec}$ ). Concomitantly, estimated maximum retinal slip velocities were nearly identical, $2.7^{\circ} / \mathrm{sec}$ in both $n \mathrm{NOS}^{-/-}$mutant and wild-type mice at this condition. The magnitude of the retinal slip velocity is thought to be the crucial factor for the adaptation of the HOKR. Furthermore, in the present and previous studies, we have consistently revealed that the adaptation of the HOKR is controlled by the flocculus in mice as well as other animals. Therefore, we suggest that the impaired adaptation of the HOKR in $n \mathrm{NOS}^{-1-}$ mutant is caused by the deficits in the flocculus but not in the precerebellar regions. However, the precise answer on the role of nNOS for adaptive control of reflex eye movements may need specio-temporal specific gene knockout mice.

Conjunctive stimulation of parallel fiber and climbing fiber inputs induces LTD of parallel fiber-Purkinje cell synapses (e.g., Ito 1989). NO is suggested to play a key role for the LTD induction in cerebellar slice (Crepel et al. 1996). Applications of L-NMMA or hemoglobin blocked long-term desensitization of glutamate receptors on Purkinje cells (Ito and Karachot 1990) and LTD of parallel fiber-Purkinje cell synapses (Crepel and Jaillard 1990; Daniel et al. 1993) in rat cerebellar slice preparation. Furthermore, endogenous NO release in the molecular layer was observed by electrochemical NO probes in parallel with the induction of cerebellar LTD (Shibuki and Okada 1991). NO activated soluble guanylate cyclase (sGC) (Stone and Marletta 1996), which increased cGMP and consequently activated cGMP-dependent protein kinase (PKG). Selective inhibitor of sGC or PKG also blocked cerebellar LTD (Boxall and Garthwaite 1996; Lev-Ram et al. 1997a) in slice preparation. Furthermore, Lev-Ram et al. (1997b) demonstrated impairment of LTD induction in cerebellar slices obtained from $n \mathrm{NOS}^{-/-}$ mutant mice by whole-cell patch clamp recording. Taken together with these observations, our results support the hypothesis that cerebellar LTD underlies the adaptation of the HOKR from both pharmacological and genetical points of view.

The adaptation of the HVOR is induced by sustained visual-vestibular interactions in almost all animal species including humans and goldfish (e.g., Ito and Nagao 1991). Several lines of experimental evidence indicate that similar
A

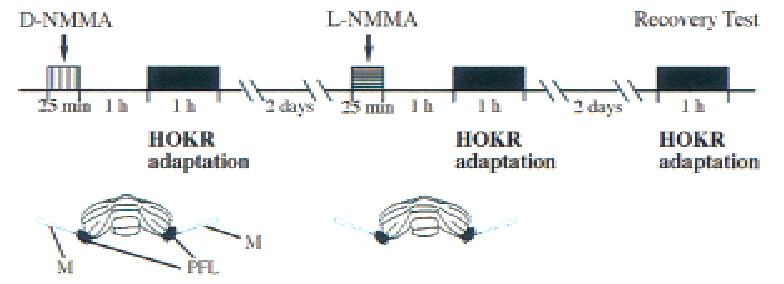

B

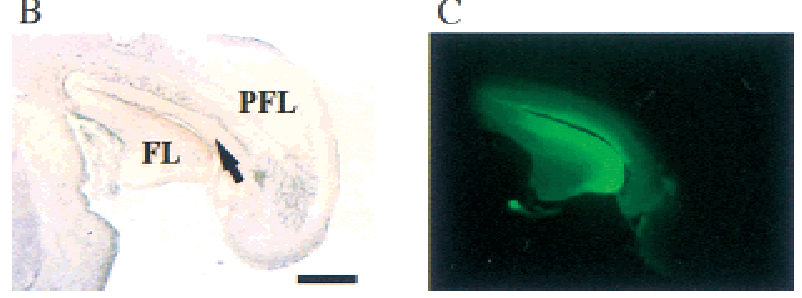

Figure 5 Experimental protocol for local application of the NO synthesis inhibitor, L-NMMA. (A) Experimental protocol for D-NMMA and L-NMMA local application. (B) Representative 10\% formalin-fixed frozen section of FITC-injected brain. (C) Diffusion of FITC seen in the adjacent section of $B$. Note that FITC diffused within flocculus and ventral paraflocculus. All chemicals were injected by $0.5 \mu \mathrm{l}$ at $0.02 \mu \mathrm{l} / \mathrm{min}$. The arrow indicates the center of injection. (FL) Flocculus; (PFL) paraflocculus; (M) micropipette. Calibration bar, $500 \mu \mathrm{m}$.

neural mechanisms are used for the adaptation of the HVOR and HOKR. First, both the adaptation of HOKR [rabbit (Nagao 1983), mouse (Katoh et al. 1998)] and HVOR [rabbit (Nagao 1983), monkey (Lisberger et al. 1984)] were abolished by lesions of the flocculus. Second, a rabbit whose HOKR was adapted by visual stimulus showed coincidentally an increase in the HVOR gain (Nagao 1989b). Third, a group of floccular Purkinje cells exhibited responses correlated well with the adaptation of both the HOKR and HVOR (Nagao 1988, 1989a). The role of NO for the adaptation of the HOKR demonstrated in this study provides additional similarity between the OKR and VOR.

\section{MATERIALS AND METHODS}

\section{Animals}

The experimental procedures and housing conditions were approved by the RIKEN Institutional Animal Care and Use Committee, along with the guideline of the National Institutes of Health (NIH). Mice lacking nNOS $\left(\mathrm{B} 6,129 \mathrm{~S}-\mathrm{Nos} 1^{\mathrm{tm} 1 \mathrm{Plh}}\right)$ were obtained from the Jackson Laboratory (Bar Harbor, ME) and maintained at RIKEN BSI Animal Care Facility. The $n \mathrm{NOS}^{-/-}$mutants were backcrossed to C57BL/6 strain obtained from Japan Clea (Tokyo, Japan) for two generations, and the heterozygotes were intercrossed to obtain mutation homozygotes. Wild-type littermates were used as a control. Mice used in the present study had a predominantly C57BL/6 genetic background (> 87\%) and were 2-3 months old. Primers used for PCR aided genotyping were 5'-AAACTCTGCAGCCGGTTCTT- 3 ' and 5 '-CCTGTCTACTGCTAATGGCT- 3 ' for wild-type allele and $5^{\prime}$-CTTGGGTGGAGAGGCTATTC- 3 ' and 5'-AGGTGAGAT-

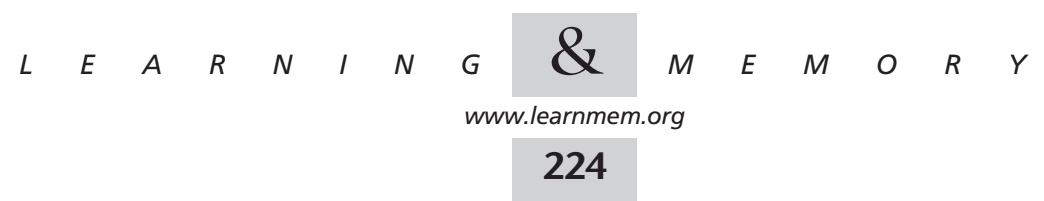


GACAGGAGATC-3' for mutant allele (neo gene cassette). PCR cycles were run at $96^{\circ} \mathrm{C}$ for $1 \mathrm{~min}, 60^{\circ} \mathrm{C}$ for $1 \mathrm{~min}$, and $72^{\circ} \mathrm{C}$ for 2 min for 35 cycles. C57BL/6 mice maintained in the facility were used for the pharmacological experiments.

\section{Operations}

Under pentobarbital anesthesia (Nacalai Tesque, Kyoto, Japan; 60 $\mathrm{mg} / \mathrm{kg}$ body weight) in aseptic conditions, a platform for fixation of the head was built on the cranial bone by using four small screws and one long bolt fixed in place by synthetic resin. For animals subjected to local applications of NO synthesis inhibitor, small holes were made bilaterally in the bones overlying the paraflocculus. No less than $48 \mathrm{hr}$ after surgery, a mouse was used for eye movement recordings.

\section{General Eye Movement Recordings}

Eye movements were recorded with an infrared TV camera for real-time eye position recording (Katoh et al. 1998). The frontal view of the right eye was monitored by the infrared CCD TV camera (SSC-M350; SONY, Tokyo, Japan) through a cold mirror. The pupil of the right eye was illuminated by an infrared light (wavelength, $900 \mathrm{~nm}$ ) -emitting diode and displayed on a 12-inch TV monitor (magnification, 55x). The area of the pupil was determined by the difference in brightness between the pupil and the iris. The real-time position of the eye was measured by calculating the central position of the left and right margin of the pupil at 50 $\mathrm{Hz}$ using a position-analyzing system (C-1170; Hamamatsu Photonics, Hamamatsu, Japan) and stored on a personal computer. The HOKR was tested by sinusoidal oscillation of the checked-pattern screen (square size $4^{\circ}$ ) by $5^{\circ}-20^{\circ}$ (peak to peak) at $0.11-0.17 \mathrm{~Hz}$ (maximum velocity, $2.6^{\circ}-10.5^{\circ} / \mathrm{sec}$ ) in light (illuminance, $200 \mathrm{~lx}$ ). The HVOR was tested by sinusoidal oscillation of the turntable in the horizontal plane by $10^{\circ}$ (peak to peak) at $0.11-0.50 \mathrm{~Hz}$ in darkness. More than six cycles of the evoked eye movements appearing to be sinusoidal waves, free from artifacts due to blinking and saccade, were averaged, and mean amplitude and phase were calculated by a modified Fourier analysis (Jastreboff 1979), as described previously (Nagao 1990). The mean effective diameter of the mouse eyeball was $3.4 \pm 0.05 \mathrm{~mm}$ (mean \pm s.E., $n=10$ ) measured by a vernier micrometer in general anesthetized mice, and gain was defined as the ratio of the peak-to-peak amplitude of eye movement versus the peak-to-peak amplitude of the turntable or screen oscillation. Phase was defined as $0^{\circ}$ when peak of eye movement matched the peak of screen oscillation in the HOKR, and when peak of eye movement was opposite to peak of turntable oscillation in the HVOR.

The adaptability of the HOKR was examined by $1 \mathrm{hr}$ of sustained screen oscillation by $10^{\circ}$ at $0.17 \mathrm{~Hz}\left(5.2^{\circ} / \mathrm{sec}\right)$ or $15^{\circ}$ at 0.17 $\mathrm{Hz}\left(7.9^{\circ} / \mathrm{sec}\right)$ in light. The HOKR was measured every $30 \mathrm{~min}$.

\section{Local Applications of L-NMMA}

The experimental timetables are schematically presented in Figure 5A. Thirteen C57BL/6 mice were used. L-NMMA and $N^{\mathrm{G}}$-monomethyl-D-arginine (D-NMMA), an inactive isomer of L-NMMA, were obtained from Nacalai Tesque. The mice were mounted on the turntable surrounded by a checked-pattern screen with the head fixed and the body loosely restrained inside a small plastic cylinder. The HOKR was examined by sinusoidal oscillation of the screen by $10^{\circ}$ and $15^{\circ}$ (peak to peak) at $0.17 \mathrm{~Hz}$ (maximum velocity, $5.2^{\circ} / \mathrm{sec}$ and $7.9^{\circ} / \mathrm{sec}$ ) in light. Then two microsyringes ( $\# 80135$; Hamilton, Sealy, TX) with fine glass pipettes connected to the needle tips and attached to standard micromanipulators were inserted to the floccular areas bilaterally through openings (1 $\mathrm{mm}$ in diameter) made on the periotic capsules, which does not include any vestibular organs. These operations did not damage the canals because any anomalies of posture, for example, a head tilt, were not observed in all of these operated mice. D-NMMA (20 mM) dissolved in PBS ( $\mathrm{pH}$ 7.4) was injected bilaterally ( $0.5 \mu \mathrm{l}$ at $0.02 \mu \mathrm{l} / \mathrm{min}$ for each side). Then, the animals were replaced in their home cages. One hour after injection, the mice were again mounted on the turntable, and the HOKR was re-examined by sinusoidal oscillation of the screen at the same velocities. Adaptability of the HOKR was tested by $1 \mathrm{hr}$ of sustained screen oscillation by $15^{\circ}$ at $0.17 \mathrm{~Hz}\left(7.9^{\circ} / \mathrm{sec}\right)$ in light. Two days later, the mice were challenged again with the measurements of the gains and phases of the HOKR by sinusoidal screen oscillation at $5.2^{\circ} / \mathrm{sec}$ and $7.9^{\circ} / \mathrm{sec}$, injection of $20 \mathrm{~mm}$ L-NMMA in the same manner as injection of D-NMMA, and the adaptability of the HOKR. After a further 2 days, the adaptability of the HOKR was reinvestigated using the same mice without injection to evaluate the possible effects of tissue damage due to injection. One of 13 mice failed to show any HOKR adaptability in both the D-NMMA injection and the recovery test. The data from this mouse was excluded from the statistics. To confirm the diffusibility of chemicals and reproducibility of the applications, $0.5 \mu \mathrm{l}$ of $1 \%$ fluorescein isothiocyanate hydrochloride (FITC; Research Organics, Cleveland, $\mathrm{OH})$ per PBS ( $\mathrm{pH} 7.4)$ was injected in the same manner as D- or L-NMMA/PBS ( $\mathrm{pH} 7.4$ ) into four $\mathrm{C} 57 \mathrm{BL} / 6$ mice. After intracardial perfusion with PBS followed by a $10 \%$ formol-PBS solution and postfixation in $10 \%$ formol-PBS solution, $40-\mu \mathrm{m}$-thick coronal sections were prepared using a conventional cryostat and observed under a UV microscope.

\section{Test of $n \mathrm{NOS}^{-/-}$Mutant Mice}

Twelve $n \mathrm{NOS}^{-1-}$ mutant mice and eight control wild-type littermates were used. The HOKR was examined by sinusoidal oscillation of the checked-pattern screen by $5^{\circ}-20^{\circ}$ at $0.11-0.17 \mathrm{~Hz}$ (maximum velocity, $2.6^{\circ}-10.5^{\circ} / \mathrm{sec}$ ) in light. Th HVOR was examined by sinusoidal oscillation of the turntable in the horizontal plane by $10^{\circ}$ at $0.11-0.50 \mathrm{~Hz}$ in darkness. To keep the size of the pupils constant, a drop of $1.0 \%$ pilocarpine hydrochloride solution (Sanpilo, Santen, Tokyo) was applied to the eye before measurements of the HVOR. The adaptability of the HOKR was tested by 1 hr of sustained screen oscillation by $10^{\circ}$ at $0.17 \mathrm{~Hz}\left(5.2^{\circ} / \mathrm{sec}\right)$ in light.

\section{ACKNOWLEDGMENTS}

We thank Dr. N. Kawai (Jichi Medical School) and all members of both laboratories for their helpful discussions. A.K. thanks Dr. K. Ito (Kyoto University) for his continuous encouragement. We thank Drs. M. Miller, H. Niki, and M. Ito for previewing the manuscript. This work was partly supported by grants from Toyota RIKEN, Japan (S.N.), and the Ministry of Health, Japan (S.I.).

The publication costs of this article were defrayed in part by payment of page charges. This article must therefore be hereby marked "advertisement" in accordance with 18 USC section 1734 solely to indicate this fact.

\section{REFERENCES}

Albus, J.S. 1971. A theory of cerebellar function. Math. Biosci. 10: 25-61. Boxall, A.R. and Garthwaite, J. 1996. Long-term depression in rat cerebellum requires both NO synthase and NO-sensitive guanylyl cyclase. Eur. J. Neurosci. 8: 2209-2212.

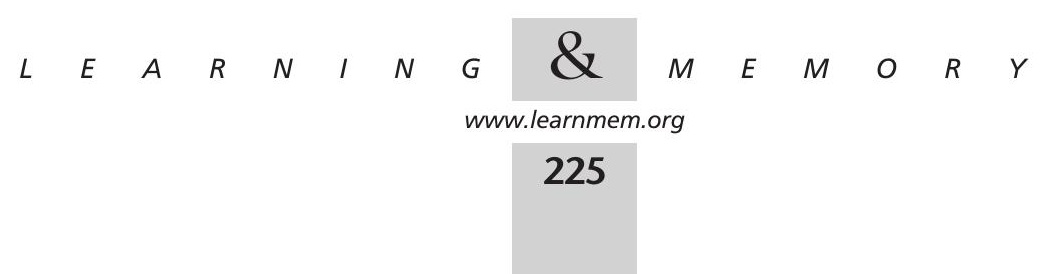


Chapman, P.F., Atkins, C.M., Allen, M.T., Haley, J.E., and Steinmetz, J.E. 1992. Inhibition of nitric oxide synthesis impairs two different forms of learning. NeuroReport 3: 567-570.

Crepel, F. and Jaillard, D. 1990. Protein kinases, nitric oxide and long-term depression of synapses in the cerebellum. NeuroReport 1: 133-136.

Crepel, F., Hemart, D. Jaillard, D., and Daniel, H. 1996. Cellular mechanisms of long-term depression in the cerebellum. Behav. Brain Sci. 19: 347-353.

Daniel, H., Hemart, D. Jaillard, D., and Crepel, F. 1993. Long-term depression requires nitric oxide and guanosine $3^{\prime}: 5^{\prime}$ cyclic monophosphate production in rat cerebellar Purkinje cells. Eur. $J$. Neurosci. 5: 1079-1082.

De Zeeuw, C.I., Hansel, C., Bian, F., Koekkoek, S.K.E., van Alphen, A.M., Linden, D.J., and Oberdick, J. 1998. Expression of a protein kinase C inhibitor in Purkinje cells blocks cerebellar LTD and adaptation of the vestibulo-ocular reflex. Neuron 20: 495-508.

Dinerman, J.L., Dawson, T.M., Schell, M.J., Snowman, A., and Snyder, S.H. 1994. Endothelial nitric oxide synthase localized to hippocampal pyramidal cells: Implications for synaptic plasticity. Proc. Natl. Acad. Sci. 91: 4214-4218.

Ekerot, C.-F. and Kano, M. 1985. Long-term depression of parallel fibre synapses following stimulation of climbing fibre. Brain Res. 342: $357-360$

Ito, M. 1984. The cerebellum and neural control. Raven Press, New York NY.

. 1989. Long-term depression. Annu. Rev. Neurosci. 12: 85-102.

Ito, M. and Karachot, L. 1990. Messengers mediating long-term desensitization in cerebellar Purkinje cells. NeuroReport 1: 129-132.

Ito, M. and Nagao, S. 1991. Comparative aspects of horizontal ocular reflexes and their cerebellar adaptive control in vertebrates. Comp. Biochem. Physiol. 98C: 221-228.

Ito, M., Sakurai, M., and Tongroach, P. 1982. Climbing fibre induced depression of both mossy fibre responsiveness and glutamate sensitivity of cerebellar Purkinje cells. J. Physiol. 324: 113-134.

Jastreboff, P.J. 1979. Evaluation and statistical judgment of neural responses to sinusoidal stimulation in cases with superimposed drift and noise. Biol. Cybern. 33: 113-120.

Katoh, A., Kitazawa, H., Itohara, S., and Nagao, S. 1998. Dynamic characteristics and adaptability of mouse vestibulo-ocular and optokinetic response eye movements and the role of the flocculo-olivary system revealed by chemical lesions. Proc. Natl. Acad. Sci. 95: 7705-7710.

Knowles, R.G. and Moncada, S. 1994. Nitric oxide synthases in mammals. Biochem. J. 298: 249-258.

Kobzik, L., Reid, M.B., Bredt, D.S., and Stamler, J.S. 1994. Nitric oxide in skeletal muscle. Nature 372: 546-548.

Lev-Ram, V., Makings, L.R., Keitz, P.F., Kao, J.P.Y., and Tsien, R.Y. 1995 Long-term depression in cerebellar Purkinje neurons results from coincidence of nitric oxide and depolarization-induced $\mathrm{Ca}^{2}+$ transients. Neuron 15: 407-415.

Lev-Ram, V., Jiang, T., Wood, J., Lawrence, D.S., and Tsien, Y. 1997a. Synergies and coincidence requirements between NO, cGMP, and $\mathrm{Ca}^{2+}$ in the induction of cerebellar long-term depression. Neuron 18: $1025-1038$.

Lev-Ram, V., Nebyelul, Z., Ellisman, M.H., Huang, P.L., and Tsien, R.Y. 1997b. Absence of cerebellar long-term depression in mice lacking neuronal nitric oxide synthase. Learn. \& Mem. 4: 169-177.

Li, J., Smith, S.S., and McElligott, J.G. 1995. Cerebellar nitric oxide is necessary for vestibulo-ocular reflex adaptation, a sensorimotor model of learning. J. Neurophysiol. 74: 489-494.

Lisberger, S.G., Miles, F.A., and Zee, D.S. 1984. Signals used to compute errors in monkey vestibuloocular reflex: Possible role of flocculus. $J$. Neurophysiol. 52: 1140-1153.

Marr, D. 1969. A theory of cerebellar cortex. J. Physiol. (Lond.) 202: $437-470$.

Murphy, S., Simmons, M.L. Agullo, L., Garcia, A., Feinstein, D.L., Galea, E., Reis, D.J., Minc-Golomb, D., and Schwartz, J.P. 1993. Synthesis of nitric oxide in CNS glial cells. Trends Neurosci. 16: 323-328.

Nagao, S. 1983. Effects of vestibulocerebellar lesions upon dynamic characteristics and adaptation of vestibulo-ocular and optokinetic responses in pigmented rabbits. Exp. Brain Res. 53: 36-46.

- 1988. Behavior of floccular Purkinje cells correlated with adaptation of horizontal optokinetic eye movement response in pigmented rabbits. Exp. Brain Res. 73: 489-497.

. 1989a. Behavior of floccular Purkinje cells correlated with adaptation of vestibulo-ocular reflex in pigmented rabbits. Exp. Brain Res. 77: 531-540.

- 1989b. Role of cerebellar flocculus in adaptive interaction between optokinetic eye movement response and vestibulo-ocular reflex in pigmented rabbits. Exp. Brain Res. 77: 541-551.

-1990. A non-invasive method for real-time eye position recording with an infrared TV-camera. Neurosci. Res. 8: 210-213.

Nagao, S. and Ito, M. 1991. Subdural application of hemoglobin to the cerebellum blocks vestibuloocular reflex adaptation. NeuroReport 2: 193-196.

Nagao, S. and Kitazawa, H. 2000. Subdural applications of NO-scavenger or NO-blocker to the cerebellum depress the adaptation of the monkey post-saccadic smooth pursuit eye movements. NeuroReport 11: 131-134.

Rodrigo, J., Springall, D.R., Uttenthal, O., Bentura, M.L., Abadia-Molina, F., Riveros-Moreno, V., Martinez-Murillo, R., Polak, J.M., and Moncada, S. 1994. Localization of nitric oxide synthase in the adult rat brain. Phil. Trans. R. Soc. Lond. B 345: 175-221.

Schmidt, H.H., Gagne, G.D., Nakane, M., Pollock, J.S., Miller, M.F., and Murad, F. 1992. Mapping of neural nitric oxide synthase in the rat suggests frequent co-localization with NADPH diaphorase but not with soluble guanylyl cyclase, and novel paraneural functions for nitrinergic signal transduction. J. Histochem. Cytochem. 40: 1439-1456.

Shibuki, K. and Okada, D. 1991. Endogenous nitric oxide release required for long-term synaptic depression in the cerebellum. Nature 349: 326-328

Stone, J.R. and Marletta, M.A. 1996. Spectral and kinetic studies on the activation of soluble guanylate cyclase by nitric oxide. Biochemistry 35: 1093-1099.

Yanagihara, D. and Kondo, I. 1996. Nitric oxide plays a key role in adaptive control of locomotion in cat. Proc. Natl. Acad. Sci. 93: 13292-13297.

Received January 12, 2000; accepted in revised form April 12, 2000.

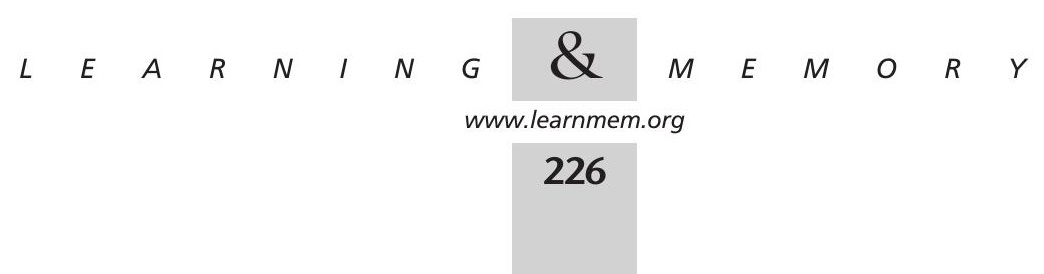




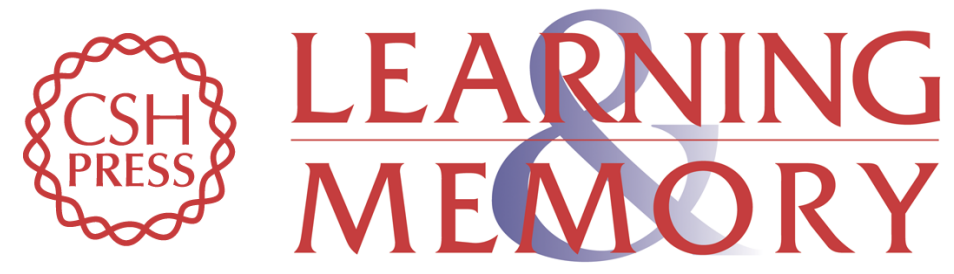

\section{Inhibition of Nitric Oxide Synthesis and Gene Knockout of Neuronal Nitric Oxide Synthase Impaired Adaptation of Mouse Optokinetic Response Eye Movements}

Akira Katoh, Hiromasa Kitazawa, Shigeyoshi Itohara, et al.

Learn. Mem. 2000, 7:

Access the most recent version at doi:10.1101//m.7.4.220

References This article cites 34 articles, 4 of which can be accessed free at: http://learnmem.cshlp.org/content/7/4/220.full.html\#ref-list-1

License

Email Alerting

Receive free email alerts when new articles cite this article - sign up in the box at the Service top right corner of the article or click here. 